\title{
A high-risk, high-grade proximal left anterior descending coronary artery stenosis in a patient with a zero calcium score
}

\author{
Adam Z Magier, Robert A Pelberg* \\ Lindner Research Center for Research and Education and The Christ Hospital, Ohio, USA
}

Received: January 21, 2016

DOI: $10.5430 /$ crim.v3n2p7

\author{
Accepted: February 25, 2016 \\ Online Published: March 4, 2016 \\ URL: http://dx.doi.org/10.5430/crim.v3n2p7
}

\begin{abstract}
A 58-year-old man with intermediate risk for obstructive coronary artery disease presents with atypical CP. A cardiac computed tomographic angiogram with calcium scoring was obtained. Despite a zero calcium score he was found to have a high risk, critical, proximal left anterior descending artery (LAD) stenosis. During the post-scan monitoring period, he developed unstable angina symptoms. He was admitted and ruled out for infarction. Invasive coronary angiography confirmed his severe LAD stenosis and a drug eluting stent was placed in the proximal LAD. This case nicely illustrates the cardiac computed tomographic angiography characteristics of a high risk coronary stenosis. In addition, it emphasizes that while a zero calcium score is associated with a good prognosis, it is not tantamount to the absence of coronary artery disease and good clinical judgment must be employed when caring for symptomatic patients, despite a zero calcium score.
\end{abstract}

Key Words: Cardiac computed tomographic angiography, Coronary artery disease, High risk coronary stenosis, Calcium score, Zero calcium score, Napkin ring sign

\section{INTRODUCTION}

Non-contrasted coronary calcium scoring (CAC) is a valuable, independent screening tool to help reclassify an intermediate cardiac risk patient into a high or low risk subset. ${ }^{[1]}$ It remains a class IIb recommendation. ${ }^{[2]}$ Patients with a zero calcium score are extremely unlikely to have coronary artery disease (CAD) ${ }^{[3,4]}$ However, their risk for obstructive CAD is not zero. ${ }^{[3,4]}$ Cardiac computed tomographic angiography (CCTA), a contrasted scan, is a state of the art diagnostic imaging modality used to evaluate patients with chest pain or other symptoms that may be attributable to CAD. Its high negative predictive value and excellent diagnostic accuracy ${ }^{[5]}$ are ideally suited for the evaluation of patients at low to in- termediate risk for CAD. CCTA also serves as a powerful prognostic tool in these patients. ${ }^{[6]}$ Furthermore, patient outcomes using CCTA as the initial imaging modality in the evaluation of chest pain have been shown to be equivalent to stress test imaging. ${ }^{[7]}$ Finally, CCTA possesses the ability, in some cases, to identify high risk, potentially unstable coronary lesions. ${ }^{[8]}$ We present a case of a man with atypical chest pain evaluated with CAC and CCTA as the initial diagnostic imaging modality. Despite a zero CAC, he was found to have a high risk, severe, proximal left anterior descending (LAD) coronary stenosis, which is a highly unlikely and clinically interesting finding in this setting.

\footnotetext{
*Correspondence: Robert A Pelberg; Email: pelbergr@ohioheart.org; Address: Lindner Research Center for Research and Education and The Christ Hospital, 11140 Montgomery Road, Cincinnati, Ohio 45249, USA.
} 


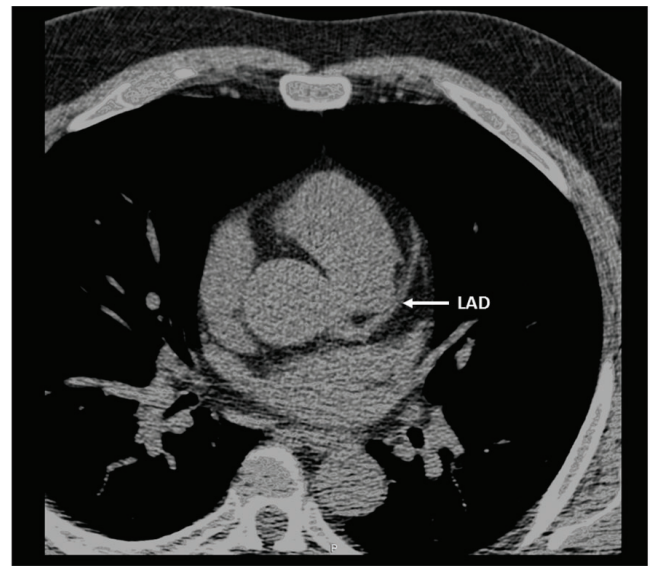

Figure 1. A non-contrasted calcium scoring image. The total calcium Agatston score was zero. In particular, note that there is no calcium in the proximal left anterior descending coronary artery (LAD)

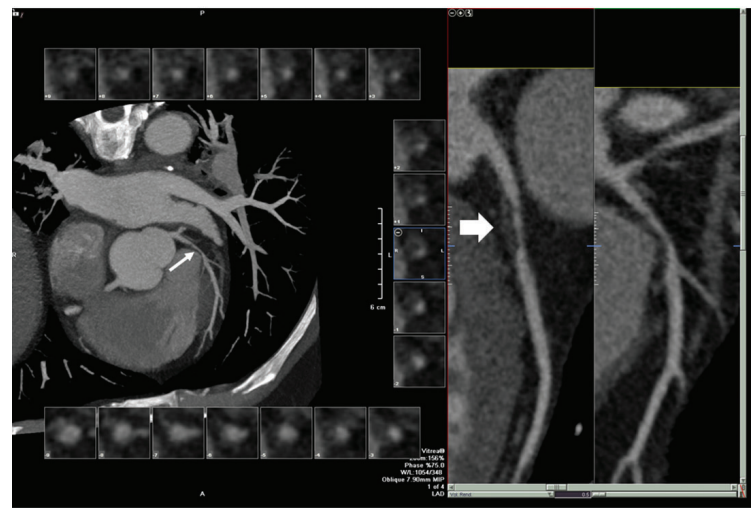

Figure 2. The left panel illustrates a maximum intensity projection image of the left anterior descending coronary artery (LAD) a the level of the critical stenosis (white arrow). The right panel is a curved multiplanar reformat of the LAD. The severe stenosis is indicated by the white block arrow. The square boxes in the left panel are cross sectional slices of the lesion at 1 millimeter increments Note. There is no calcium within the lesion.
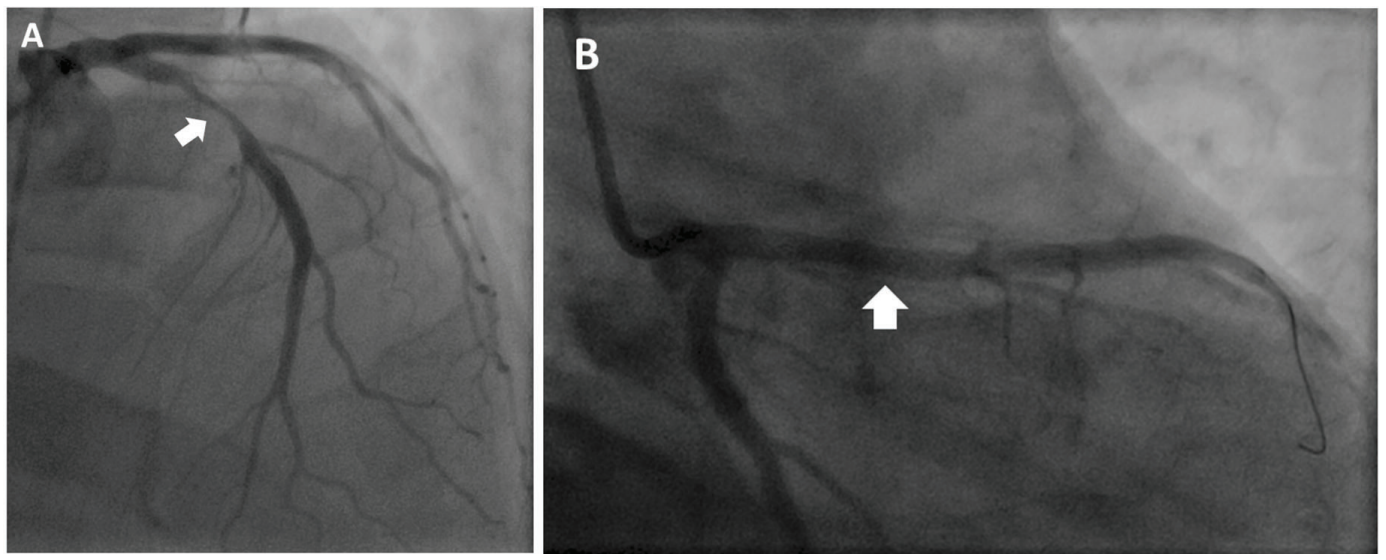

Figure 3. Panels A and B depict the proximal left anterior descending coronary artery stenosis (block arrows) before and after stenting respectively. The lesion closely resembles that seen on the coronary computed tomography image Note. There is no calcium seen on the fluoroscopic image in panel A.

\section{Case presentation}

A 58-year-old man with hypertension presented to the office with atypical chest pain. He described his chest pain as a nagging ache in the center of his chest without radiation. The ache was constant and unrelenting for 24 to 48 hours at a time. It did not radiate and there were no associated symptoms of shortness of breath, diaphoresis or nausea. The pain was unpredictable and not related to exertion. His family history was significant for three sisters and four brothers who suffered myocardial infarctions before the age of 50 . He was a nonsmoker with no history of diabetes mellitus or hyperlipidemia. We determined he was intermediate risk (16\% 10-year-risk using the Framingham Risk Calculator) for obstructive coronary artery disease. While his symptoms were markedly atypical, his intermediate cardiac risk and strong family history of early CAD obligated an evaluation for obstructive $\mathrm{CAD}$. Because of its high negative predictive value for the identification of $\mathrm{CAD},{ }^{[5]}$ its ability to reclassify intermediate risk patients into high and low cardiac risk, ${ }^{[3,4]}$ and its ability to identify other, non-cardiac causes of chest pain, a CCTA with CAC was ordered. Another advantage to this approach over stress test imaging is the ability of CCTA to identify subclinical CAD, not identifiable by stress testing. This added value of CCTA adds credence to secondary CAD prevention recommendations if subclinical CAD is identified.

The calcium score was zero (see Figure 1). The CCTA revealed normal right coronary and circumflex arteries. The 
LAD, however, demonstrated a high grade, proximal, noncalcified stenosis with high risk features (see Figure 2), including positive remodeling of the artery, a napkin-ring sign and high plaque burden. ${ }^{\left[{ }^{8,9]}\right.}$ Because the patient complained of typical resting angina during his post-CCTA monitoring period, he was admitted to the hospital with a suspected acute coronary syndrome. His cardiac enzymes were negative but he continued to have intermittent resting angina. An invasive coronary angiogram confirmed the high grade proximal, noncalcified LAD lesion seen by CCTA (see Figure 3). A drug eluting stent was placed in the proximal LAD (see Figure 3) and the patient was discharged the next day without complication. At his 2-week follow up office visit the patient was chest pain free and feeling well.

\section{Discussion}

It is our opinion that the patient's initial, outpatient symptoms were not caused by his coronary stenosis. However, the more typical, resting symptoms after the CCTA most certainly were consistent with unstable angina, related to the proximal LAD lesion. We believe that his proximal LAD lesion was initially a stable lesion and serendipitously became unstable after the CCTA exam. This hypothesis is supported by the high risk CCTA characteristics of this lesion, rendering it a potentially unstable stenosis. The timing of an acute coronary syndrome related to a particular unstable lesion is not clinically predictable. We do not feel that this patient's ACS was in any way caused by the CCTA itself. The excellent safety record of CCTA is well documented in the literature. ${ }^{[5,7]}$

Coronary calcium scoring is highly predictive of prognosis and cardiac risk. ${ }^{[1]}$ While a zero calcium score may be reassuring, it is well recognized that the absence of coronary calcium is not equivalent to the absence of coronary artery disease. ${ }^{[3,4]}$ Significant coronary stenoses are found in approximately $2.5 \%$ of patient's with zero calcium and are located most often in the proximal LAD. ${ }^{[3,4]}$ High risk coronary stenoses are characterized by low attenuation, large plaque volumes, positive remodeling, spotty calcifications and the napkin-ring sign, ${ }^{[8,9]}$ most of which were observed in our patient's proximal LAD lesion (see Figure 4). These high risk features rendered this lesion a possible vulnerable plaque and may account for the onset of the unstable acute coronary syndrome suffered immediately after the CCTA; the timing of the unstable angina was likely coincidental. This case highlights the importance good clinical judgment in symptomatic patients, despite a zero calcium score. A zero calcium score alone may not exclude stable or unstable angina in a patient with suggestive symptoms. In addition, calcium scoring cannot always be the sole measure for excluding significant coronary artery atherosclerosis. Published by Sciedu Press
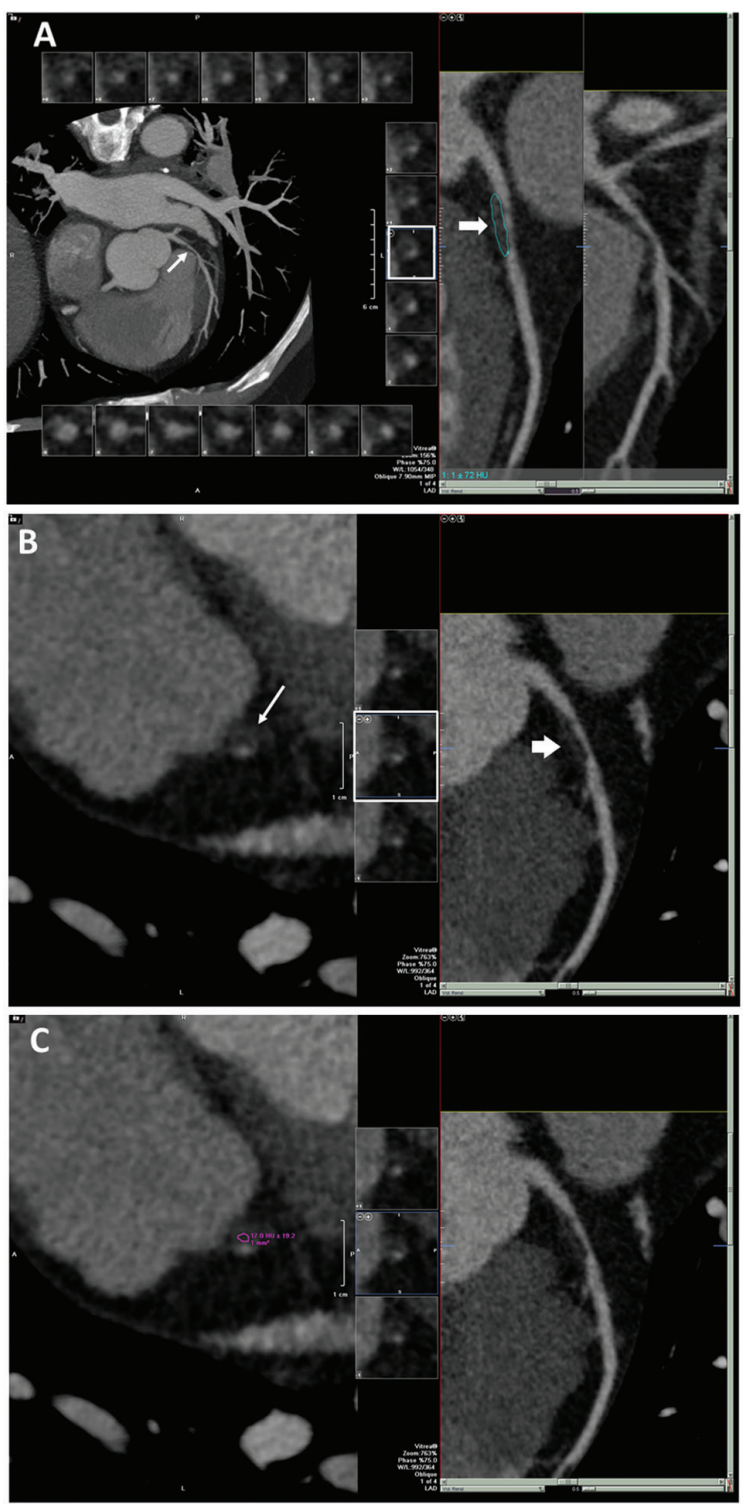

Figure 4. Panels A, B and C are multiplanar (MPR) and curved multiplanar (cMPR) reformats of the proximal left anterior descending stenosis and highlight the high risk features of this lesion. The white arrows on the left sides of panels A and B highlight the lesion location in the proximal LAD in the long axis (panel A) and cross section (panel B). The blue outline on the cMPR in panel A encircles the entire eccentric plaque and demonstrates its large plaque burden and low attenuation (an average of 72 Hounsfield units [HU]). Note the positive remodeling of the lesion. Both panels $\mathrm{A}$ and $\mathrm{B}$ illustrate the napkin ring feature (white box in panels A and B and white arrow in panel B). Panel C depicts the very low attenuation within the napkin-ring of the lesion (17 HU). The white block arrows point to the lesion on the cMPR images in panels $\mathrm{A}$ and $\mathrm{B}$

\section{CONFliCtS OF INTEREST Disclosure}

The authors have declared no conflicts of interest. 


\section{REFERENCES}

[1] Budoff MJ, Shaw LJ, Liu ST, et al. Long-term prognosis associated with coronary calcification. Observations from a registry of 25,253 patients. J Am Coll Cardiol. 2007; 49(18): 1860-1870. PMid:17481445 http://dx.doi.org/10.1016/j.jacc.2006.10.079

[2] Greenland P, Alpert JS, Beller GA, et al. 2010 ACCF/AHA Guideline for Assessment of Cardiovascular Risk in Asymptomatic Adults: Executive Summary.

[3] Büyükterzi M, Türkvatan A, Büyükterzi Z. Frequency and extent of coronary atherosclerotic plaques in patients with a coronary artery calcium score of zero: assessment with CT angiography. Diagn Interv Radiol. 2013; 19(2): 111-8. PMid:23271579

[4] Morita H, Fujimoto S, Kondo T, et al. Prevalence of computed tomographic angiography-verified high-risk plaques and significant luminal stenosis in patients with zero coronary calcium score. Int $\mathbf{J}$ Cardiol. 2012; 158(2): 272-8. PMid:21420188 http://dx.doi.o rg/10.1016/j.ijcard.2011.02.052

[5] Meijboom BW, Meijs MFL, Schuijf JD, et al. Diagnostic accuracy of 64-slice computed tomography coronary angiography: a prospective, multicenter, multivendor study. J Am Coll Cardiol. 2008; 52(25):
2135-2144. PMid:19095130 http://dx.doi.org/10.1016/j·j acc. 2008.08 .058

[6] Kalra DK, Heo R, Valenti V, et al. Role of computed tomography for diagnosis and risk stratification of patients with suspected or known coronary artery disease. Arterioscler Thromb Vasc Biol. 2014; 34(6): 1144-1154. PMid:24723554 http://dx.doi.org/10.1161/ATV BAHA. 113. 302074

[7] Douglas PS, Hoffmann U, Patel MR, et al. Outcomes of anatomical versus functional testing for coronary artery disease. New Engl J Med. 2015; 372(14): 1291-1300. PMid:25773919 http: //dx.doi.org/10.1056/NEJMoa1415516

[8] Pflederer T, Marwan M, Schepis T, et al. Characterization of culprit lesions in acute coronary syndromes using coronary dualsource CT angiography. Atherosclerosis. 2010; 211(2): 43744. PMid:20189568 http://dx.doi.org/10.1016/j .atheros clerosis.2010.02.001

[9] Papadopoulou SL, Neefjes LA, Garcia-Garcia HM, et al. Natural history of coronary atherosclerosis by multislice computed tomography. JACC Cardiovasc Imaging. 2012; 5(3 Suppl): S2837. PMid:22421228 http://dx.doi.org/10.1016/j.jcmg. 20 12.01 .009 